\title{
An Active Tracking System Using IEEE 802.15.4-Based Ultrasonic Sensor Devices
}

\author{
Shinyoung Yi and Hojung Cha \\ Department of Computer Science, Yonsei University \\ Seodaemun-gu, Shinchon-dong 134, Seoul 120-749, Korea \\ \{mainwork, hjcha\}@cs.yonsei.ac.kr
}

\begin{abstract}
Utilization of an ultrasound tracking system in wireless sensor networks is a well-known technique with low-cost and high-accuracy advantages in an indoor environment. In this paper, we present the implementation of an active tracking system based on an ultrasonic sensing device using the IEEE 802.15.4 compatible radio. IEEE 802.15.4 is used in wireless sensor networks because of its low power consumption and high bit-rate. Many of the technical issues for actual deployment of the system in an indoor environment are herein analyzed and solved.
\end{abstract}

\section{Introduction}

A Location Based Service (LBS) technique that uses Global Positioning System (GPS) satellites or cellular networks has been implemented and deployed for tracking systems in the outdoor environment. However, with a GPS device it is not possible to receive the RF satellite signal inside a building, and an indoor location system requires higher accuracy than GPS. Therefore, location techniques using GPS devices are inappropriate for an indoor location system. Many techniques for an appropriate indoor location system have recently been proposed. There are those using infrared[1], wireless LAN[2, 3], and both radio and ultrasound[4, 5, 6]. An indoor location system should guarantee high accuracy, low cost, and secure user privacy[4]. A location system using synchronized RF signals and ultrasonic pulses in wireless sensor networks is a known technique that satisfies these requirements.

Existing location techniques using ultrasound suffer an inherent limitation of system performance and user privacy due to their old-fashioned radio modules with low bit-rates or use of wired networks. An IEEE 802.15.4 compatible radio[7] with a high bit-rate and low power consumption is widely used in wireless sensor networks. In this paper we propose a tracking system with an ultrasound-based location technique, employing a widely-used IEEE 802.15.4 compatible radio to improve upon previous localization techniques. The proposed technique guarantees high accuracy and user privacy through distributed processing with an active tracking model[8]. We have implemented the system in a real indoor environment, and analyzed the technical limitations and run-time problems. The technical issues surrounding the operation of the proposed ultrasonic tracking system are determined and solved through experiment and analysis. We have validated the performance by actual experiment, and the proposed technique is found to be applicable to an indoor location system. 
The remainder of the paper is organized as follows: Section 2 introduces techniques for an ultrasound-based location system. Sections 3 and 4 present technical issues surrounding implementation of a tracking system using ultrasound and an IEEE 802.15.4 compatible radio. The performance of an implemented tracking system is evaluated in Section 5, and Section 6 concludes the paper.

\section{Related Work}

An ultrasonic location system is categorized as an active or a passive model[8], based on whether or not the target node transmits the RF and ultrasonic signals on its own. In the active model, a target node transmits RF and ultrasonic signals to receiving nodes to estimate distance; however, in the passive model, the target node measures distances to the beacon nodes by receiving the RF and ultrasonic signals from them.

The Cricket Location System[4] is a well-known passive implementation of an ultrasound-based location system. In it, a target node receives the RF and ultrasonic signals, which the fixed beacon nodes knowing their absolute coordinates transmit periodically, and calculates the distance to the beacon nodes by TDoA (Time Difference of Arrival) of the RF signal and ultrasonic pulse. More than three distances between a target node and beacon nodes enable the calculation of the location of a target node by applying trilateration. The Cricket system uses B-MAC[9] in TinyOS $[10,11]$, and the CC1000 transceiver[12], and has the advantage of accurate distance measurement through easy synchronization control of the RF signal and ultrasonic pulse. However, the CC1000 transceiver consumes a significant amount of power and has a low bit-rate[13]. The Cricket system adopts the passive method, and shows lower accuracy in continuously-moving target tracking than does the active method, guaranteeing the user privacy through the distributed method and light communication load between sender and receiver[8]. The proposed technique in this paper guarantees both accuracy in tracking and user privacy by adopting the active method, and processes the location in the distributed method.

The Active Bat[5] is distinguished from the Cricket in that a sender called Bat transmits the synchronized RF signal and ultrasonic pulse actively. In the Bat system, the receiving node that receives both $\mathrm{RF}$ and ultrasonic signals measures the distance and sends the distance information to a host wired to it. The final location of the target node is calculated by trilateration at the host. The Active Bat uses a $433 \mathrm{MHz}$ radio channel for synchronization with the ultrasonic signal. Receivers send the distance information to the wire-connected host, and the location is calculated by a centralized method at the host. Since the distance information is communicated through wire, high reliability of the information is guaranteed. However, it has problems: initial installation of communication cables is required and a centralized method user privacy guarantee is difficult to achieve. The proposed system in our research, however, guarantees user privacy as it calculates the location of the target node at the sender side through the distributed method $[4,8]$. 


\section{Active Tracking System}

Fig. 1 illustrates the overall structure of an active tracking system. The system is constructed with a mobile node and backbone nodes. The Mobile node is a moving node, and backbone nodes are set up to measure the distance between the mobile and backbone nodes. The mobile node sends synchronized radio signals and ultrasonic pulses periodically. The radio signal includes the node ID and sequence information of the mobile node. When a backbone node receives a radio signal, it perceives the subsequent ultrasonic pulse and measures the time difference. The backbone nodes compute the distance between the mobile node and themselves by the time difference and send the distance information to the mobile node. The mobile node receives more than three pieces of distance information from the backbone nodes and calculates its location on a 2D coordinates system by trilateration. Since this process is performed at every beaconing period, it is possible to track the location of the moving mobile node.

Because our system is an active architecture, wherein the mobile node actively sends the beacon messages, the backbone nodes obtain concurrent distance information. This feature of the architecture renders the tracking accurate[8]. However, active architecture has radio channel overhead compared to passive architecture, since more than three backbone nodes send distance information to a mobile node simultaneously. Furthermore, packet loss may occur on the mobile node while it receives distance information from backbone nodes concurrently. We provide a solution for these problems in Section 4. The calculated coordinates of the mobile node are sent to the closest backbone node. The data is sent to a host through a routing method among the backbone nodes and displayed by a Graphical User Interface (GUI) application on the host.

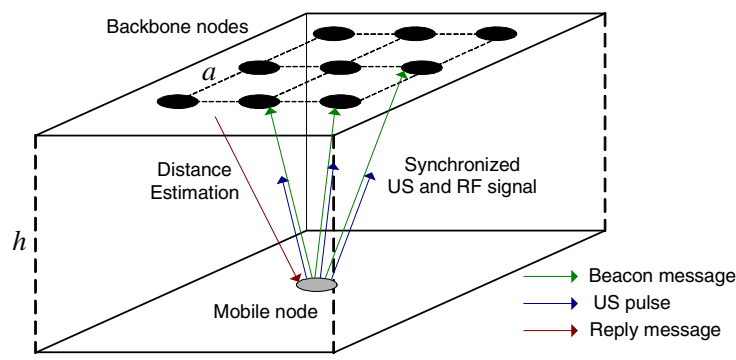

Fig. 1. Active tracking system

\section{Implementation Issues}

Ultrasound-based tracking using the IEEE 802.15.4 radio has different technical problems from previous schemes. The tracking system needs to be reliable in real deployment in indoor environments. In the following subsections, we analyze these 
problems and describe solutions to implement a tracking system applicable to an actual environment.

\subsection{Synchronizing US Pulse with RF Signal}

To implement an ultrasound-based tracking system, we developed an ultrasonic sensor hardware module that sends or detects a $40 \mathrm{Kh}$ ultrasonic pulse. We also developed a wireless sensor mote (Fig. 6(b)) operated with the ultrasonic sensor. The mote is similar to Telos[14], which uses a MSP430 F1611[15] micro controller, a CC2420, and an IEEE 802.15.4 compatible radio transceiver chip[16].

The radio stack of the Cricket system is implemented as part of the software application. This enables control of the synchronization time of the radio signal and ultrasonic pulse on the software level. By controlling the data packet from its header to the $\mathrm{CRC}$, the Cricket system maintains regular time intervals between radio signal and ultrasonic pulse. It is correct to regard the required time for ultrasonic pulse arrival as the arrival time difference between RF signal and ultrasonic pulse.

Since the CC2420 radio stack is implemented on PHY and operated on the hardware level, it is difficult to control the point of packet transmission and reception time on the software level. The system is required to synchronize the ultrasonic pulse after the packet transmission is complete. However, if the times required for data transmission are irregular, the measured arrival time difference between RF and ultrasound would include some errors. If we synchronize the ultrasonic pulse with the time before the packets are sent, the arrival time difference could be converted to the ultrasound transmit time precisely. In this case, however, the receiver cannot distinguish the synchronized radio signal from radio signals for other purposes. To apply the system to an actual environment, a solution to this problem is necessary. In this paper, we synchronize the arrival time of an ultrasonic pulse with the packet destination address checking time in the MAC layer. Fig. 2 shows the differences in synchronizing time between the Cricket and our system.

\subsection{Trilateration}

A mobile node calculates its position through trilateration after receiving more than three pieces of distance information from backbone nodes. Since the system is designed for indoor application, the height of the space is considered constant. Fig. 3 shows the mechanism by which to calculate the position of a point by distance information from three positions. Suppose there are three points with known positions. These positions are expressed as $\left(x_{1}, y_{1}, h\right),\left(x_{2}, y_{2}, h\right)$, and $\left(x_{3}, y_{3}, h\right)$ where $h$ is the height of the $3 \mathrm{D}$ space. $\hat{d}_{1}, \hat{d}_{2}$ and $\hat{d}_{3}$ represent the distance estimations from each known point to unknown 2D coordinates $(x, y)$ where $z=0 \cdot d_{i}$, the distance on the $\mathrm{x}-\mathrm{y}$ plane, is expressed as Equation 1.

$$
d_{i}=\sqrt{\hat{d}_{i}^{2}-h^{2}}, i=1,2,3
$$



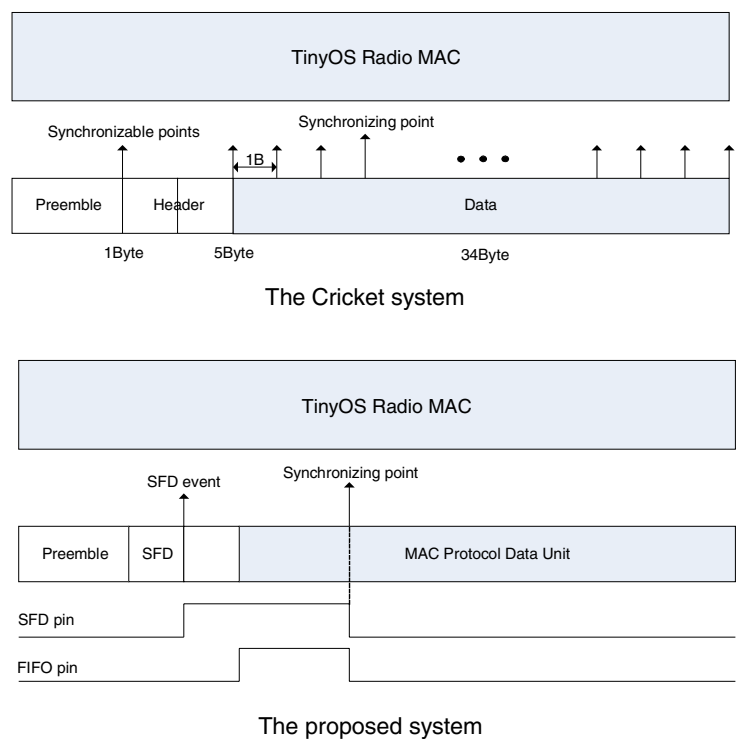

Fig. 2. Comparison of our system's synchronization point with that of the Cricket system

Trilateration is not accurate when the distance values include comparably large errors. In our system, this problem occurs more frequently when the mobile node receives more than three pieces of distance information. To reduce the error in trilateration, we choose the three smallest distance values among the received distances since smaller distances include fewer errors. Fig. 4 shows the distance measurement error according to the angle of sender and receiver. The distance error and the measuring degree are directly proportional. In real deployment, the angle of a mobile and a backbone node is larger when the distance increases. In other words, significant errors would be included in long-distance estimates. Hence, trilateration with short distances calculates accurate positions in the system.

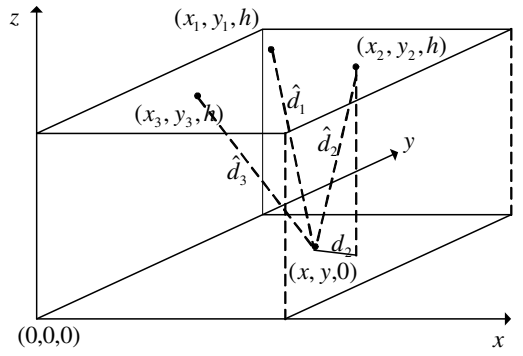

Fig. 3. Trilateration in mapping $3 \mathrm{D}$ to $2 \mathrm{D}$

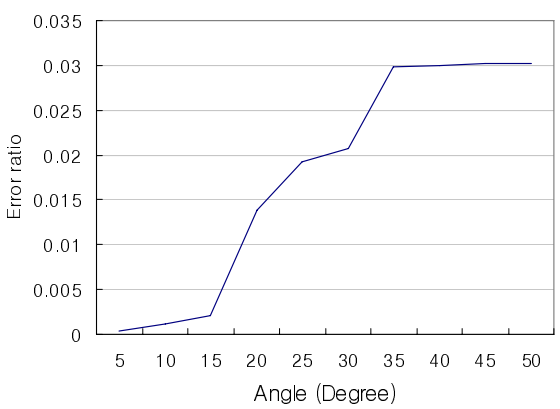

Fig. 4. Distance error according to degrees 


\subsection{Distance Error Filtering}

Distance measurement generally includes errors caused by obstacles and other environmental factors. Since reliable distance measurement is directly related to the performance of the tracking system, preventing these errors is an important issue in the system. However, the errors usually occur when measuring angles and distance values are large. We use two methods to solve this problem.

Height of the indoor space, $h$, is the minimum distance the backbone node can measure. In other words, the minimum distance value, MIN_DISTANCE, is determined as the constant value $h$. When the backbone node measures a distance longer than MIN_DISTANCE, it implies that the backbone node and the mobile node are not in a straight line. It also means the distance value possibly includes distance error. We multiply the distance information by a constant $\alpha$ to revise the data. The modified $d_{\text {new }}$ is expressed as Equation 2. The constant $\alpha$ is proportional to the difference between $\hat{d}$ and MIN_DISTANCE. We obtained the constant $\alpha$ through real environment experiments. In an environment with $235 \mathrm{~cm}$ of height and $150 \mathrm{~cm}$ of backbone node grid size, the value of the constant $\alpha$ is 0.98 .

$$
d_{\text {new }}=\alpha \cdot \hat{d}
$$

Fig. 5(a) shows the ultrasound range, in different positions, of a mobile node in the sensor field. In conditions with ultrasound range $r$ and grid size $a$, at least three backbone nodes will detect the ultrasound wherever the mobile node is located. Circle A shows the situation wherein a minimum number of backbone nodes will detect the ultrasound pulse. Circle B represents the situation wherein a maximum number of backbone nodes will detect the ultrasonic pulse. The maximum distance estimation, MAX_DISTANCE, is calculated through Equation 3. In our system, backbone nodes send back the distance information to the mobile node when $d_{\text {new }}$ is greater than MIN_DISTANCE and less than MAX_DISTANCE. This method reduces the error of measurement and prevents unnecessary packet transmissions.

$$
\text { MAX_DISTANCE }=\sqrt{h^{2}+2 a^{2}}
$$

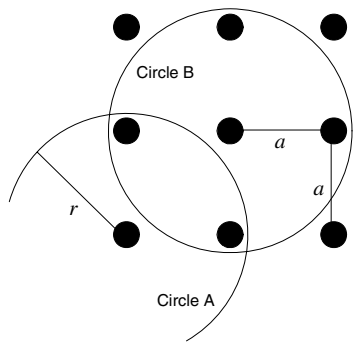

(a) Ultrasonic coverage

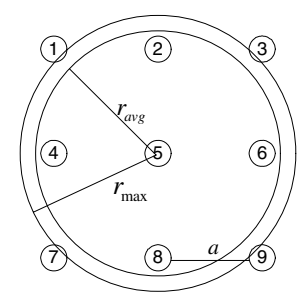

(b) Maximum and average range of US pulse

Fig. 5. Ultrasonic range 


\subsection{Radio Reliability}

When the height of the indoor space is $235 \mathrm{~cm}$ and backbone nodes are deployed on a grid with $150 \mathrm{~cm}$ intervals, a maximum of nine backbone nodes respond to the ultrasound pulse of a mobile node. Fig. 5(b) shows the situation when nine backbone nodes are in the range of an ultrasonic pulse. Although the distance estimation would be filtered by the method we describe in Section 4.3, there is a situation in which all nine backbone nodes send distance information to the mobile node. The distance measurement error problem with nodes 1, 3, 7, and 9 is solved by sorting the distances and using only three small distance values. However, there could be a radio problem when a mobile node tries to receive simultaneous response messages. There is a possibility of losing packets when simultaneous radio packets arrive at the mobile node. To solve this problem, each backbone node is set for random delay before it sends the distance information to the mobile node. We have also designed the backbone nodes to retransmit the distance message for reliability of the system.

\section{Evaluation}

Fig. 6 shows the experimental testbed using nine backbone nodes, a gateway node, a mobile node, and a line-tracking motor car. We investigated the tracking performance of our proposal system through the GUI application shown in Fig. 6(b).

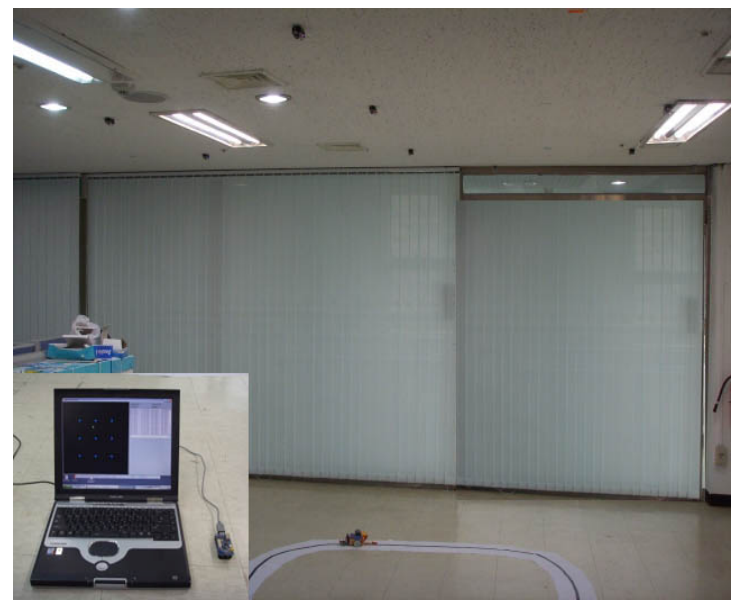

(a) System deployment
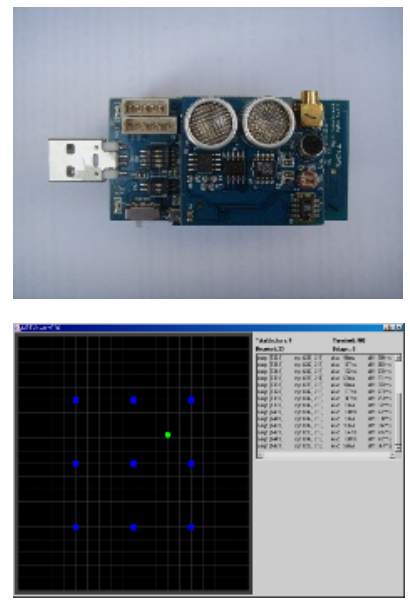

(b) Sensor mote with an ultrasonic module and the GUI

Fig. 6. The ultrasonic location system using an IEEE 802.15.4 radio

In order to deploy the system successfully, more than three of the backbone nodes to the ceiling should be able to detect the ultrasonic pulse robustly. The ultrasonic sensor device we developed could sense the ultrasonic pulse within a distance of 11 meters in a straight line. When the distance is not straight, the range of detectable 
ultrasonic pulses is in a circular arc with an angle of 120 degrees and a radius of 230 $\mathrm{cm}$. In addition to a reliable detection range, both the arrangement and the density of the backbone nodes have important effects on robust localization when the backbone nodes are deployed in a ceiling. In the case that the backbone nodes within the fixed range are arranged densely, many backbone nodes are able to sense an ultrasonic pulse simultaneously. It may cause packet loss to transmit too many distance estimations to a mobile node, and a narrow arrangement would cost more. Sparse arrangement of the backbone nodes may cause a mobile node not to receive enough distance estimations because of obstructions or other RF signals. Receiving enough distance estimations is the prerequisite for successful positioning. Hence when the backbone nodes are attached to a ceiling, arrangement and density should be considered. We deployed nine backbone nodes in the $150 \mathrm{~cm}$ by $150 \mathrm{~cm}$ grid topology.

Fig. 7 describes the result of tracking a moving motor car at a speed of 0.3 meters per second. The mobile node attached to the motor car broadcasts the beacon message at $500 \mathrm{~ms}$ intervals, and the series of positions are displayed at the same intervals. Our tracking system shows good performance that computed coordinates with an average $20 \mathrm{~cm}$ error. The sources of error are considered to be in measurement of height and distance.

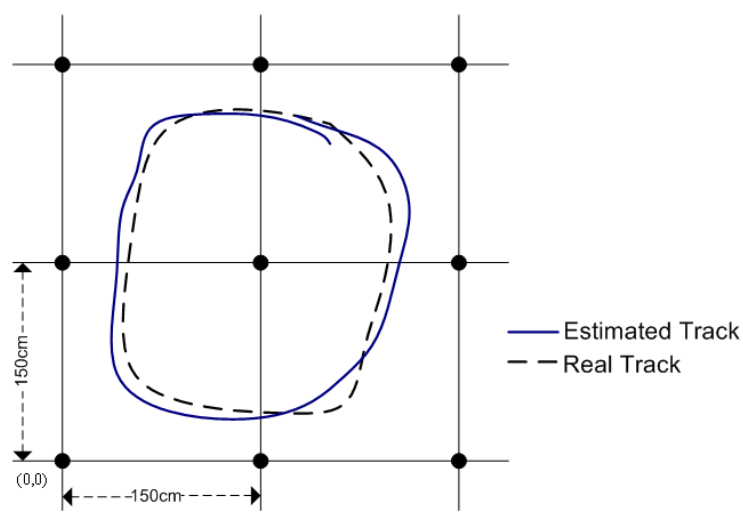

Fig. 7. Tracking the moving node

Fig. 8 shows the positioning difference between conditions when both a distance error filtering policy and radio reliability technique are applied and otherwise. The maximum localization error arises when a mobile node is located at the identical $x-y$ coordinates of backbone node 5 in Fig. 5(b). The error can be minimized when there is a mobile node in the center of the 4 one-hop neighbor nodes constructing a quadrangle. We examined the difference of the location result between the maximum error position, $(150,150)$, and the minimum error position, $(250,70)$. We sampled 50 coordinates computing at each location. Figs. 8(a) and 8(c) show the result of no policies, and Figs. 8(b) and 8(c) represent the estimated positions by the proposed system including distance error filtering and radio reliability policy. There is no significant error found in performing localization by adopting the proposed schemes. 


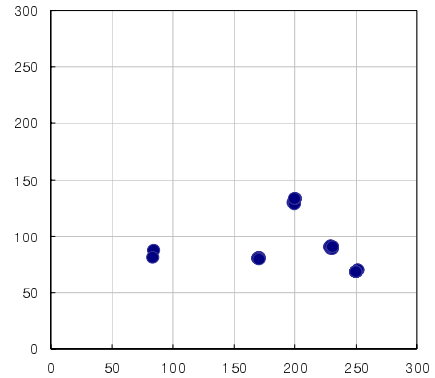

(a) Without error filtering and radio reliability: position $(250,70)$

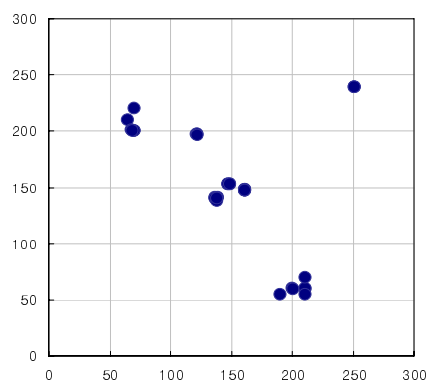

(c) Without error filtering and radio reliability: position $(150,150)$

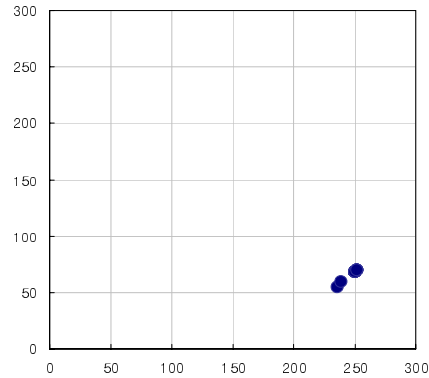

(b) Error filtering and radio reliability: position $(250,70)$

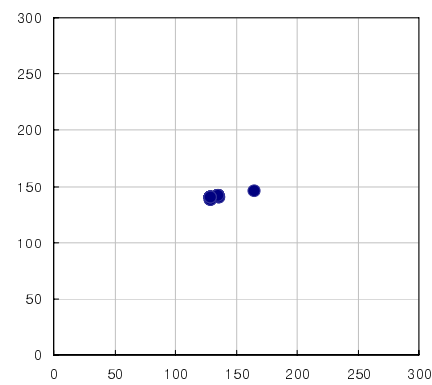

(d) Error filtering and radio reliability : position $(150,150)$

Fig. 8. Error distribution of computed coordinates

\section{Conclusion}

In this paper, we have presented an active tracking system using the IEEE 802.15.4 compatible radio. Our system, with the active method, guarantees user privacy and accurate tracking of a moving device, and therefore can be deployed in a real indoor environment. We validated the proposed system as a real application by deploying and testing it in our laboratory. Our contribution for deploying an ultrasound-based active tracking system is as follows: The arrangement of backbone nodes should be decided in consideration of the height from sender to receiver, and the reachable range of an ultrasonic pulse. Adjusting the measurement error is required because an error of distance estimation increases according to the increment of the angle between ultrasound transmitter and receiver. A protocol that guarantees robustness is required in an active system wherein a mobile node receives both RF signals and ultrasonic pulses at once. The proposed active tracking system including these schemes has actually been implemented and analyzed. This system may be utilized for such indoor location-aware services as object tracking and human navigation. 


\section{Acknowledgements}

This work was supported by the National Research Laboratory (NRL) program of the Korean Science and Engineering Foundation (2005-01352) and the ITRC Program (MMRC) of IITA, Korea.

\section{References}

1. R. Want, A. Hopper, V. Falcao, and J. Gibbons. The Active Badge Location System. In ACM Transactions on Information Systems, January 1992.

2. P. Bahl and V. N. Padmanabhan. RADAR: An In-Building RF-Based User Location and Tracking System. In INFOCOM 2000, Vol. 2, pp. 775-784, March 2000.

3. M. A. Youssef, A. Agrawala, and A. U. Shankar. WLAN Location Determination via Clustering and Probability Distributions. In Proceedings of the First IEEE International Conference on Pervasive Computing and Communications (PerCom 2003), pp. 143-152, March 2003.

4. N. B. Priyantha, A. Chakraborty, and H. Balakrishnan. The Cricket Location-Support System. In $6^{\text {th }}$ ACM International Conference on Mobile Computing and Networking (Mobicom 00), August 2000.

5. A. Harter, A. Hopper, P. Steggles, A. Ward, and P. Webster. The Anatomy of a ConetxtAware Application. In 5th ACM International Conference on Mobile Computing and Networking (Mobicom 99), pp. 59-68, August 1999.

6. M. Hazas and A. Ward. A Novel Broadband Ultrasonic Location System. In Proceedings of 4th International Conference on Ubiquitous Computing (UbiComp 2002), 2002.

7. IEEE Standard for Information Technology: 802.15.4: Wireless Medium Access Control and Physical Layer Specifications for Low-Rate Wireless Personal Area Networks. 2003 http://standards.ieee.org/getieee802/download/802.15.4-2003.pdf

8. A. Smith, H. Balakrishnan, and M. Goraczko. Tracking Moving Devices with the Cricket Location System. In 2nd International Conference on Mobile Systems, Applications and Services (Mobisys 2004), June 2004.

9. J. Polastre, J. Hill, and D. Culler. Versatile Low Power Media Access for Wireless Sensor Networks, In ACM SenSys 2004, November 2004.

10. http://www.tinyos.net

11. J. Hill, R. Szewczyk, A. Woo, S. Hollar, D. Culler, and K. Pister. System Architecture Directions for Network Sensors, In Proceedings of ASPLOS 2000, November 2000.

12. http://www.chipcon.com/files/CC1000_Data_Sheet_2_3.pdf

13. J. Polastre, R. Szewczyk, and D. Culler. Telos: Enabling Ultra-Low Power Wireless Research. In Proceedings of IPSN/SPOTS, April 2005.

14. http://www.moteiv.com

15. http://www.ti.com

16. http://www.chipcon.com/files/CC2420_Data_Sheet_1_3.pdf 